\title{
Summary and agreement statement of the 2nd International Conference on Concussion in Sport, Prague 2004
}

\author{
P McCrory, K Johnston, W Meeuwisse, M Aubry, R Cantu, J Dvorak, T Graf-Baumann, J Kelly, \\ M Lovell, P Schamasch
}

See end of article for

authors' affiliations

.......................

Correspondence to:

Associate Professor

McCrory, PO Box 93

Shoreham, Victoria 3916

Australia; paulmccr@

bigpond.net.au

Accepted 7 February 2005
Br J Sports Med 2005;39:196-204. doi: 10.1136/bjsm.2005.018614

In November 2001, the 1st International Symposium on Concussion in Sport was held in Vienna, Austria to provide recommendations for the improvement of safety and health of athletes who suffer concussive injuries in ice hockey, football (soccer), and other sports. The 2nd International Symposium on Concussion in Sport was organised by the same group and held in Prague, Czech Republic in November 2004. It resulted in a revision and update of the Vienna consensus recommendations, which are presented here.
$\mathrm{T}$ his paper is a revision and update of the Vienna consensus recommendations developed after the lst International Symposium on Concussion in Sport. ${ }^{1}$ The Prague agreement statement is designed to build on the principles outlined in the original Vienna document and to develop further conceptual understanding of this problem. This document is developed for use by doctors, therapists, health professionals, coaches, and other people involved in the care of injured athletes, whether at the recreational, elite, or professional level.

\section{BACKGROUND PERSPECTIVE}

In November 2001, the 1st International Symposium on Concussion in Sport was held in Vienna, Austria. This meeting was organised by the International Ice Hockey Federation (IIHF) in partnership with the Federation Internationale de Football (FIFA) and the International Olympic Committee Medical Commission (IOC). As part of the resulting mandate for the future, the need for leadership and updates was identified. To meet that mandate, the 2nd International Symposium on Concussion in Sport was organised by the same group and held in Prague, Czech Republic in November 2004.

The original aims of the symposia were to provide recommendations for the improvement of safety and health of athletes who suffer concussive injuries in ice hockey, football (soccer), and other sports. To this end a range of experts were invited to both meetings in order to address specific issues of epidemiology, basic and clinical science, injury grading systems, cognitive assessment, new research methods, protective equipment, management, prevention, and long term outcome. At the conclusion of the initial conference, a small group of experts were given a mandate by the conference delegates and organising bodies to draft a document describing the agreement position reached by those in attendance at that meeting. That document was co-published in the British Journal of Sports Medicine, Clinical Journal of Sport Medicine, and Physician and Sportsmedicine. ${ }^{1}$

The wider interest base resulting from the first meeting and document was reflected by the expanded representation. New groups at the second meeting included trauma surgeons, sport psychologists, and others. This same group has produced the current document as an update of the original Vienna consensus document and includes a sideline assessment form with a pocket sized summary card for use by clinicians.

This protocol represents a work in progress, and, as with all other recommendations or proposals, it must be updated as new information is added to the current state of the literature and understanding of this injury.

\section{BACKGROUND ISSUES}

\section{Definition of concussion}

Over 35 years ago, the Committee on Head Injury Nomenclature of the Congress of Neurological Surgeons proposed a "consensus" definition of concussion. ${ }^{23}$ This definition was recognised as having a number of limitations in accounting for the common symptoms of concussion. In the Vienna document, a revised consensus definition was proposed as follows: "Sports concussion is defined as a complex pathophysiological process affecting the brain, induced by traumatic biomechanical forces". Several common features that incorporate clinical, pathological, and biomechanical injury constructs that may be used in defining the nature of a concussive head injury include the following.

(1) Concussion may be caused by a direct blow to the head, face, neck, or elsewhere on the body with an "impulsive" force transmitted to the head.

(2) Concussion typically results in the rapid onset of short lived impairment of neurological function that resolves spontaneously.

(3) Concussion may result in neuropathological changes, but the acute clinical symptoms largely reflect a functional disturbance rather than structural injury.

(4) Concussion results in a graded set of clinical syndromes that may or may not involve loss of consciousness. Resolution of the clinical and cognitive symptoms typically follows a sequential course.

(5) Concussion is typically associated with grossly normal structural neuroimaging studies.

No changes were made to the definition by the Prague Group beyond noting that in some cases post-concussive symptoms may be prolonged or persistent. 


\section{Pathophysiological basis of concussion}

At this time, there is no existing animal or other experimental model that accurately reflects a sporting concussive injury. It is noted that, in experimental models, of more severe injury a complex cascade of biochemical, metabolic, and gene expression changes occur. ${ }^{4}$ Whether similar metabolic changes occur in sports concussion, however, remains speculative at this time. ${ }^{5}$

\section{Concussion grading scales}

The Vienna recommendation that injury grading scales be abandoned in favour of combined measures of recovery in order to determine injury severity (and/or prognosis) and hence individually guide return to play decisions received continued support.

It was also noted that concussion severity can only be determined in retrospect after all concussion symptoms have cleared, the neurological examination is normal, and cognitive function has returned to baseline. ${ }^{6}$ There is limited published evidence that concussion injury severity correlates with the number and duration of acute concussion signs and symptoms and/or degree of impairment on neuropsychological testing. ${ }^{7-12}$ The development of validated injury severity scales continues in the published literature. ${ }^{13}$

\section{Subtypes of concussion}

One of the issues speculated on at the Vienna conference was whether concussion represents a unitary phenomenon with a linear spectrum of injury severity or whether different concussion subtypes exist. These subtypes may represent differences in clinical manifestations (confusion, memory problems, loss of consciousness), anatomical localisation (such as cerebral versus brainstem), biomechanical impact (rotational versus linear force), genetic phenotype (apolipoprotein epsilon 4 (ApoE4) positive versus ApoE4 negative), neuropathological change (structural injury versus no structural injury), or an as yet undefined difference. These factors may operate independently or interact with each other. It is clear that the variations in clinical outcome with the same impact force require a more sophisticated approach to the understanding of this phenomenon than currently available. ${ }^{14}$

\section{Significance of loss of consciousness}

The traditional approach to severe traumatic brain injury using loss of consciousness as the primary measure of injury severity has acknowledged limitations in assessing the severity of sporting concussive injury. Findings in this field describe association of loss of consciousness with specific early deficits but does not necessarily imply severity. ${ }^{13}{ }^{15}$ As such the presence of loss of consciousness as a symptom would not necessarily classify the concussion as complex (see below).

\section{Significance of amnesia}

There is renewed interest in the role of post-traumatic amnesia and its role as a surrogate measure of injury severity. ${ }^{13} 16$ Published evidence suggests that the nature, burden, and duration of the clinical post-concussive symptoms may be more important than the presence or duration of amnesia alone. ${ }^{815}$ Further it must be noted that retrograde amnesia varies with the time of measurement after the injury and hence is poorly reflective of injury severity. ${ }^{18} 19$

\section{Paediatric concussive injury}

The general recommendations outlined in the Vienna document were originally designed for the management of adult sporting concussion. Agreement was reached, however, that identified those recommendations as relevant and useful to management of children as well. In broad terms it was felt that the recommendations should be applicable to children (defined as 5-18 years of age) whereby children should not be allowed to return to play or training until clinically completely symptom free. In addition, the concept of "cognitive rest" was introduced with special reference to a child's need to limit exertion with activities of daily living and to limit scholastic activities while symptomatic. There was also a recognition by the group that additional research is needed to better clarify the potential differences between adults and children with regard to recovery from injury and to develop cognitive assessment tools that better evaluate the younger athlete.

Formal cognitive assessment is currently problematic until late teen years because of the continuing cognitive maturation that occurs during this period, which, in turn, makes the utility of comparison with either the person's own baseline performance or population norms limited. ${ }^{20}$

Because of the different physiological response during childhood to head trauma, a conservative return to play approach is recommended. It may be appropriate to extend the amount of time of asymptomatic rest and/or the length of the graded exertion in children and adolescents. Future research is needed in this area.

\section{A NEW CLASSIFICATION OF CONCUSSION IN SPORT}

Historically, concussions have been classified with a number of different grading systems. In the Vienna Statement, this approach was abandoned. One of the key developments by the Prague Group is the understanding that concussion may be categorised for management purposes as either simple or complex.

\section{Simple concussion}

In simple concussion, an athlete suffers an injury that progressively resolves without complication over 7-10 days. In such cases, apart from limiting playing or training while symptomatic, no further intervention is required during the period of recovery, and the athlete typically resumes sport without further problem. Formal neuropsychological screening does not play a role in these circumstances, although mental status screening should be a part of the assessment of all concussed athletes. Simple concussion represents the most common form of this injury and can be appropriately managed by primary care physicians or by certified athletic trainers working under medical supervision. ${ }^{21}$ The cornerstone of management is rest until all symptoms resolve and then a graded programme of exertion before return to sport. All concussions mandate evaluation by a medical doctor.

\section{Complex concussion}

Complex concussion encompasses cases where athletes suffer persistent symptoms (including persistent symptom recurrence with exertion), specific sequelae (such as concussive convulsions), prolonged loss of consciousness (more than one minute), or prolonged cognitive impairment after the injury. This group may also include athletes who suffer multiple concussions over time or where repeated concussions occur with progressively less impact force. In this group, there may be additional management considerations beyond simple return to play advice. Formal neuropsychological testing and other investigations should be considered in complex concussions. It is envisaged that such athletes would be managed in a multidisciplinary manner by doctors with specific expertise in the management of concussive injury such as a sport medicine doctor with experience in concussion, sports neurologist, or neurosurgeon. 


\section{CLINICAL ISSUES}

\section{Pre-participation physical examination}

Recognising the importance of concussion history, and appreciating the fact that many athletes will not recognise all the concussions they may have suffered in the past, a detailed concussion history is of value. ${ }^{22-25}$ Such a history may identify athletes that fit into the "complex" category outlined above and provides an opportunity for the doctor to educate the athlete about the significance of concussive injury.

A structured concussion history should include specific questions as to previous symptoms of a concussion, not just perceived number of past concussions. It is also worth noting that dependence on the recall of concussive injuries by team mates or coaches has been shown to be unreliable. ${ }^{22}$ The clinical history should also include information about all previous head, face, or neck injuries, as these may have clinical relevance to the present injury. It is worth emphasising that, with maxillofacial and neck injuries, co-existent concussive injuries may be missed unless specifically assessed. Specific questions pertaining to disproportionate impact versus symptom severity matching may alert the clinician to a progressively increasing vulnerability to injury.

As part of the clinical history, it is advised that details on protective equipment used at the time of injury be sought, both for recent and remote injuries. The benefit of this approach allows modification and optimisation of protective behaviour and an opportunity for education.

It is specifically recommended that:

(1) both a baseline cognitive assessment (such as the Prague SCAT test in the absence of computerised neuropsychological testing) and symptom score is performed as part of the pre-participation evaluation;

(2) although formal baseline neuropsychological screening may be beyond the resources of many sports or indivifdual athletes, it is recommended that, in organised high risk sports, consideration be given to having cognitive evaluation regardless of the age or level of performance.

\section{Signs and symptoms of acute concussion}

The suspected diagnosis of sports concussion made on the sideline is applicable to both medical and non-medical personnel and can include clinical symptoms, physical signs, cognitive impairment, and/or loss of consciousness.

If any one of the following symptoms or problems is present, a head injury should be suspected and appropriate management instituted. These will be summarised on the sideline concussion assessment tool (SCAT) that accompanies this document (fig l).

\section{(a) Cognitive features (see below)}

- Unaware of period, opposition, score of game

- Confusion

- Amnesia

- Loss of consciousness

(b) Typical symptoms (see SCAT (fig 1) for standard symptom scale); other symptoms such as a subjective feeling of slowness and fatigue after an impact may indicate that a concussion has occurred or has not fully resolved. ${ }^{26}$

- Headache or pressure in the head

- Balance problems or dizziness

- Nausea
- Feeling "dinged", "foggy", stunned, or "dazed"

- Visual problems-for example, seeing stars or flashing lights, double vision

- Hearing problems-for example, ringing in the ears

- Irritability or emotional changes

(c) Physical signs

- Loss of consciousness/impaired conscious state

- Poor coordination or balance

- Concussive convulsion/impact seizure

- Gait unsteadiness/loss of balance

- Slow to answer questions or follow directions

- Easily distracted, poor concentration

- Displaying inappropriate emotions-for example, laughing, crying

- Vomiting

- Vacant stare/glassy eyed

- Slurred speech

- Personality changes

- Inappropriate playing behaviour-for example, running in the wrong direction

- Significantly decreased playing ability

Sideline evaluation of cognitive function is an essential component in the assessment of this injury. Brief neuropsychological test batteries that assess attention and memory function have been shown to be practical and effective. Such tests include the Maddocks questions ${ }^{27}$ and the Standardised assessment of concussion..$^{28}$ It is worth noting that standard orientation questions-for example, time, place, personhave been shown to be unreliable in the sporting situation when compared with memory assessment. ${ }^{27} 29$

It is recognised, however, that abbreviated testing paradigms are designed for rapid concussion evaluation on the sidelines and are not meant to replace comprehensive neuropsychological testing, which is sensitive enough to detect subtle deficits that may exist beyond the acute episode, nor should they be used as a stand alone tool for the ongoing management of sports concussions. It should also be recognised that the appearance of symptoms may be delayed several hours after a concussive episode.

\section{Convulsive and motor phenomena}

A variety of acute motor phenomena-for example, tonic posturing-or convulsive movements may accompany a concussion. $^{30}{ }^{31}$ Although dramatic, these clinical features are generally benign and require no specific management beyond the standard treatment for the underlying concussive injury.

\section{Development of the sport concussion assessment tool} (SCAT)

Figure 1 outlines the SCAT. The intent was to create a standardised tool that could be used for patient education as well as for physician assessment of sports concussion. The SCAT was developed by combining the following existing tools into a new standardised tool:

(1) Sideline evaluation for concussion. ${ }^{28} 29$

(2) Management of concussion sports palm card; American Academy of Neurology and the Brain Injury Association. ${ }^{32}$

(3) Standardised assessment of concussion. ${ }^{33}$

(4) Sideline concussion check; UPMC, Thinksafe, Sports Medicine New Zealand Inc and the Brain Injury Association. 
This tool represents a standardized method of evaluating people after concussion in sport. This Tool has been produced as part of the Summary and Agreement Statement of the Second International Symposium on Concussion in Sport, Prague 2004

Sports concussion is defined as a complex pathophysiological process affecting the brain, induced by traumatic biomechanical forces. Several common features that incorporate clinical, pathological and biomechanical injury constructs that may be utilized in defining the nature of a concussive head injury include:

1. Concussion may be caused either by a direct blow to the head, face, neck or elsewhere on the body with an 'impulsive' force transmitted to the head.

2. Concussion typically results in the rapid onset of short-lived impairment of neurological function that resolves spontaneously.

3. Concussion may result in neuropathological changes but the acute clinical symptoms largely reflect a functional disturbance rather than structural injury.

4. Concussion results in a graded set of clinical syndromes that may or may not involve loss of consciousness. Resolution of the clinical and cognitive symptoms typically follows a sequential course.

5. Concussion is typically associated with grossly normal structural neuroimaging studies.

Post Concussion Symptoms

Ask the athlete to score themselves based on how they feel now. It is recognized that a low score may be normal for some athletes, but clinical judgment should be exercised to determine if a change in symptoms has occurred following the suspected concussion event.

It should be recognized that the reporting of symptoms may not be entirely reliable. This may be due to the effects of a concussion or because the athlete's passionate desire to return to competition outweighs their natural inclination to give an honest response.

If possible, ask someone who knows the athlete well about changes in affect, personality, behavior, etc.

Remember, concussion should be suspected in the presence of ANY ONE or more of the following:

- Symptoms (such as headache), or

- Signs (such as loss of consciousness), or - Memory problems

Any athlete with a suspected concussion should be monitored for deterioration (i.e., should not be left alone) and should not drive a motor vehicle.

For more information see the "Summary and Agreement Statement of the Second International Symposium on Concussion in Sport" in the: Clinical Journal of Sport Medicine 2005; $x x(x x)$ : xxx-x British Journal of Sports Medicine 2005; $x x(x x)$ : $x x x-x$ Neurosurgery 2005; ; xx(xx): xxx-x

Physician and Sportsmedicine 2005; $x x(x x)$ : $x x x-x$ This tool may be copied for distribution to teams, groups and organizations.
ตีเ

\section{$\infty$}

菑IIHF

The SCAT Card

(Sport Concussion Assessment Tool)

Athlete Information

What is a concussion? A concussion is a disturbance in the function of the brain caused by a direct or indirect force to the head. It results in a variety of symptoms (like those listed below) and may, or may not, involve memory problems or loss of consciousness.

How do you feel? You should score yourself on the following symptoms, based on how you feel now.

\begin{tabular}{|c|c|c|c|c|c|c|c|}
\hline \multicolumn{8}{|c|}{ Post Concussion Symptom Scale } \\
\hline \multirow[b]{2}{*}{ Headache } & \multicolumn{2}{|c|}{ None } & \multicolumn{3}{|c|}{ Moderate } & \multicolumn{2}{|c|}{ Severe } \\
\hline & 0 & 1 & 2 & 3 & 4 & 5 & 6 \\
\hline "Pressure in head" & 0 & 1 & 2 & 3 & 4 & 5 & 6 \\
\hline Neck Pain & 0 & 1 & 2 & 3 & 4 & 5 & 6 \\
\hline Balance problems or dizzy & 0 & 1 & 2 & 3 & 4 & 5 & 6 \\
\hline Nausea or vomiting & 0 & 1 & 2 & 3 & 4 & 5 & 6 \\
\hline Vision problems & 0 & 1 & 2 & 3 & 4 & 5 & 6 \\
\hline Hearing problems / ringing & 0 & 1 & 2 & 3 & 4 & 5 & 6 \\
\hline "Don't feel right" & 0 & 1 & 2 & 3 & 4 & 5 & 6 \\
\hline Feeling "dinged" or "dazed" & 0 & 1 & 2 & 3 & 4 & 5 & 6 \\
\hline Confusion & 0 & 1 & 2 & 3 & 4 & 5 & 6 \\
\hline Feeling slowed down & 0 & 1 & 2 & 3 & 4 & 5 & 6 \\
\hline Feeling like "in a fog" & 0 & 1 & 2 & 3 & 4 & 5 & 6 \\
\hline Drowsiness & 0 & 1 & 2 & 3 & 4 & 5 & 6 \\
\hline Fatigue or low energy & 0 & 1 & 2 & 3 & 4 & 5 & 6 \\
\hline More emotional than usual & 0 & 1 & 2 & 3 & 4 & 5 & 6 \\
\hline Irritability & 0 & 1 & 2 & 3 & 4 & 5 & 6 \\
\hline Difficulty concentrating & 0 & 1 & 2 & 3 & 4 & 5 & 6 \\
\hline Difficulty remembering & $\underline{0}$ & 1 & 2 & 3 & 4 & 5 & 6 \\
\hline
\end{tabular}

(follow up symptoms only)

Sadness

Nervous or Anxious

Trouble falling asleep

Sleeping more than usual

Sensitivity to light

Sensitivity to noise

Other:

$\begin{array}{lllllll}0 & 1 & 2 & 3 & 4 & 5 & 6 \\ 0 & 1 & 2 & 3 & 4 & 5 & 6 \\ 0 & 1 & 2 & 3 & 4 & 5 & 6 \\ 0 & 1 & 2 & 3 & 4 & 5 & 6 \\ 0 & 1 & 2 & 3 & 4 & 5 & 6 \\ 0 & 1 & 2 & 3 & 4 & 5 & 6 \\ 0 & 1 & 2 & 3 & 4 & 5 & 6\end{array}$

What should I do?

Any athlete suspected of having a concussion should be removed from play, and then seek medical evaluation.

Signs to watch for:

Problems could arise over the first $24-48$ hours. You should not be left alone and must go to a hospital at once if you:

- Have a headache that gets worse

- Are very drowsy or can't be awakened (woken up)

- Can't recognize people or places

- Have repeated vomiting

- Behave unusually or seem confused; are very irritable

- Have seizures (arms and legs jerk uncontrollably)

- Have weak or numb arms or legs

- $\quad$ Are unsteady on your feet; have slurred speech

Remember, it is better to be safe. Consult your doctor after a suspected concussion.

What can I expect?

Concussion typically results in the rapid onset of short-lived impairment that resolves spontaneously over time. You can expect that you will be told to rest until you are fully recovered (that means resting your body and your mind). Then, your doctor will likely advise that you go through a gradual increase in exercise over several days (or longer) before returning to sport.

Figure 1 Sport concussion assessment tool (SCAT). 
B

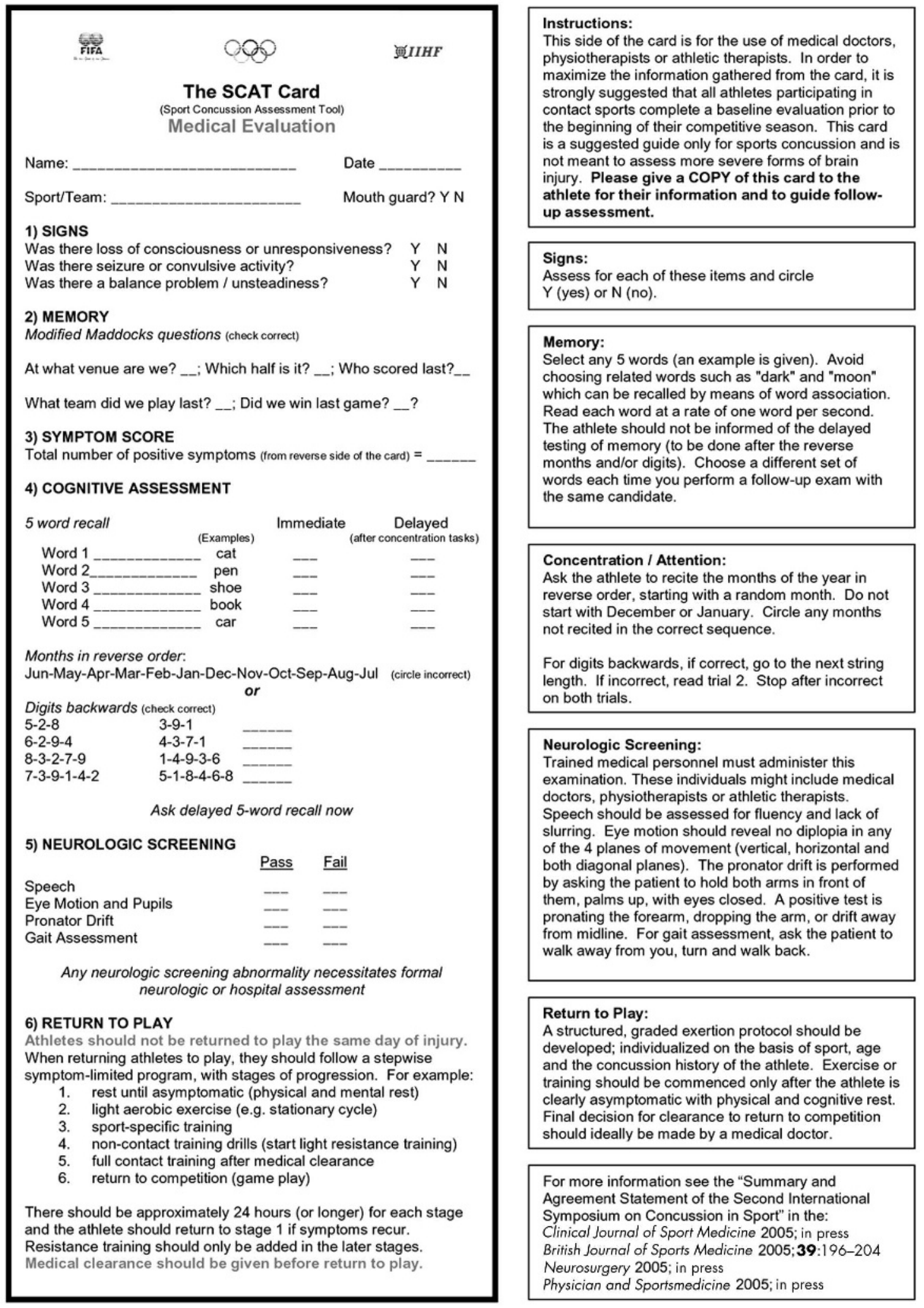

Figure 1 Continued. 
(5) McGill abbreviated concussion evaluation (ACE) (unpublished).

(6) National Hockey League physician evaluation form (unpublished).

(7) The UK Jockey Club assessment of concussion. ${ }^{34}$

(8) Maddocks questions. ${ }^{27}$

The authors gave input through a process of collaboration and iterative review. The SCAT was evaluated for face and content validity on the basis of scientific literature ${ }^{35}$ and clinical experience of the authors. The memory questions, specifically, were modified from the validated Maddocks questions to make these questions less football-specific. ${ }^{27}$

\section{INVESTIGATIONAL ISSUES \\ Neuropsychological assessment after concussion}

The application of neuropsychological testing in concussion has been shown to be of value and continues to contribute significant information in concussion evaluation. ${ }^{10113637}$ It has been shown that cognitive recovery may precede or follow clinical symptom resolution, suggesting that the assessment of cognitive function should be an important component in any return to play protocol. ${ }^{12}$ It must be emphasised, however, that neuropsychological assessment should not be the sole basis of a return to play decision but rather be seen as an aid to the clinical decision making. Although neuropsychological screening may be performed or interpreted by other healthcare professionals, the final return to play decision should remain a medical one in which a multidisciplinary approach has been taken.

Neuropsychological testing should not be performed while the athlete is symptomatic because it adds nothing to return to play decisions, and it may contaminate the testing process by allowing practice effects to confound the results. In certain cases, however, serial follow up after the injury is valuable, both as a means to encourage athlete compliance and for comparison purposes.

Over-riding principles common to all neuropsychological test batteries is the need for and benefit of baseline testing before injury and serial follow up. Recent work with computerised platforms, however, suggests that performance variability may be a key measure for acute concussion diagnosis even in the absence of a baseline test. This strategy is currently the subject of research. Inherent problems with most neuropsychological tests include the normal ranges, sensitivity and specificity of tests, and practice or learning effect, as well as the observation that players may return to baseline while still symptomatic. ${ }^{36}$ Computerised testing using infinitely variable test paradigms may overcome some of these concerns. Computerised testing also has the logistical advantage that the tests may be administered by the team doctor (or be web based) rather than requiring a neuropsychologist for a formal assessment. The strengths and weaknesses of such testing have been reviewed. ${ }^{37}$

It is recommended that neuropsychological testing remain one of the cornerstones of concussion evaluation in complex concussion. It is not currently regarded as important in the evaluation of simple concussion. Although this modality contributes significantly to both the understanding of the injury and management of the individual athlete, neuropsychological testing should not be the sole basis of management decisions, either for continued time out or return to play decisions.

\section{Objective balance assessment}

Balance testing, either with computerised platforms or clinical assessment, may offer additional information in concussed athletes and may be used as a part of the overall concussion management strategy, particularly where symptoms or signs indicate a balance component. ${ }^{38}$

\section{Neuroimaging}

It was recognised in the Vienna agreement document that conventional structural neuroimaging is usually normal in concussive injury. Given that caveat, the following suggestions are made. Computed tomography (or, where available, magnetic resonance imaging) of the brain contributes little to concussion evaluation, but should be used whenever suspicion of an intracerebral structural lesion exists. Examples of such situations may include prolonged disturbance of conscious state, focal neurological deficit, or worsening symptoms.

Newer structural magnetic resonance imaging modalities, including gradient echo, perfusion, and diffusion weighted imaging, have greater sensitivity for structural abnormalities, but the lack of published studies as well as the absence of pre-injury neuroimaging data limits the usefulness of this approach in clinical management at the present time.

In addition, the predictive value of various magnetic resonance imaging abnormalities that may be incidentally discovered is not established. Although there have been some compelling findings with promising new functional imaging technologies-for example, positron emission tomography (PET), single photon emission computed tomography (SPECT), and functional magnetic resonance imaging (fMRI) - they are still at early stages of development. ${ }^{39-41}$

Although neuroimaging may play a part in the assessment of complex sports concussions or more severe brain injury, it is not essential for simple concussive injury.

\section{Genetic testing}

Genotyping has been shown to be of benefit in traumatic brain injury. Published studies have shown that ApoE4 is a risk factor for adverse outcome after all levels of brain injury. ${ }^{42-48}$ Similarly ApoE4 has been shown to be a risk factor for the development of chronic traumatic encephalopathy in boxers. ${ }^{49}$ The significance of ApoE4 in sports concussion risk or injury outcome is unclear. Other published studies have noted the association of a particular calcium subunit gene abnormality with brain swelling after minor head trauma. ${ }^{50}$ Although still in the early stages of understanding, routine genetic screening cannot be recommended at the present time. Furthermore, doctors are urged to be mindful of the ethical implications of such testing.

\section{Experimental concussion assessment modalities}

Different electrophysiological recording techniques such as evoked response potential and electroencephalogram have shown reproducible abnormalities in the post-concussive state. ${ }^{51-53}$ However, not all studies reliably differentiated concussed athletes from controls. ${ }^{54-57}$ The clinical significance of these changes remains to be established.

In addition, biochemical serum markers of brain injury (including S-100b, NSE, MBP, GFAP) have been proposed as means by which cellular damage may be detected if present. $^{5859}$ However, there is currently not sufficient evidence to justify the use of these markers clinically.

\section{CONCUSSION MANAGEMENT}

\section{Acute injury}

When a player shows any symptoms or signs of a concussion, the following should be applied.

(1) The player should not be allowed to return to play in the current game or practice. 
(2) The player should not be left alone, and regular monitoring for deterioration is essential over the initial few hours after injury.

(3) The player should be medically evaluated after the injury.

(4) Return to play must follow a medically supervised stepwise process.

A player should never return to play while symptomatic. "When in doubt, sit them out!"

\section{Return to play protocol}

As described above, most injuries will be simple concussions, and such injuries recover spontaneously over several days. In these situations, it is expected that an athlete will proceed rapidly through the stepwise return to play strategy. ${ }^{60}$

During this period of recovery in the first few days after an injury, it is important to emphasise to the athlete that physical and cognitive rest is required. Activities that require concentration and attention may exacerbate the symptoms and as a result delay recovery.

The return to play after a concussion follows a stepwise process:

(1) No activity, complete rest. Once asymptomatic, proceed to level 2 .

(2) Light aerobic exercise such as walking or stationary cycling, no resistance training.

(3) Sport specific exercise-for example, skating in hockey, running in soccer; progressive addition of resistance training at steps 3 or 4 .

(4) Non-contact training drills.

(5) Full contact training after medical clearance.

(6) Game play.

With this stepwise progression, the athlete should continue to proceed to the next level if asymptomatic at the current level. If any post-concussion symptoms occur, the patient should drop back to the previous asymptomatic level and try to progress again after 24 hours.

In cases of complex concussion, the rehabilitation will be more prolonged, and return to play advice will be more circumspect. It is envisaged that complex cases should be managed by doctors with a specific expertise in the management of such injuries.

An additional consideration in return to play is that concussed athletes should not only be symptom-free but also should not be taking any pharmacological agents/drugs that may affect or modify the symptoms of concussion. If antidepressant treatment is started during the management of a complex concussion, the decision to return to play while still receiving such medication must be considered carefully by the clinician concerned (see below).

In professional sport, where there are team doctors experienced in concussion management as well as access to immediate-that is, sideline-neurocognitive assessment, return to play management is often more rapid, but it must still follow the same basic principles, namely full clinical and cognitive recovery before consideration of return to play.

\section{Role of pharmacological treatment}

Pharmacological treatment in sports concussion may be applied in two distinct situations: (a) management of specific symptoms-for example, sleep disturbance, anxiety-in complex concussion; $(b)$ to modify the underlying pathophysiology of the condition with the aim of shortening the duration of the concussion symptomatology. ${ }^{61}$
In broad terms, this approach to management should be only considered in complex sports concussions and by clinicians experienced in concussion management.

\section{Sports psychology}

In addition, sport psychology approaches may have potential application in this injury, particularly in complex concussion. ${ }^{62}$ Care givers are also encouraged to evaluate the concussed athlete for affective symptoms such as depression as these may be common in concussion. ${ }^{60}$

\section{OTHER ISSUES}

\section{Prevention}

There is no clinical evidence that currently available protective equipment will prevent concussion. In certain sports, protective equipment may prevent other forms of head injury which may be an important issue for those sports.

Consideration of rule changes-for example, no head checking in ice hockey-to reduce the head injury rate may be appropriate where a clear-cut mechanism is implicated in a particular sport. Similarly, rule enforcement is a critical aspect of such approaches, and referees play an important role.

An important consideration in the use of protective equipment is the concept of risk compensation. ${ }^{63}$ This is where the use of protective equipment results in behavioural change such as the adoption of more dangerous playing techniques, which can result in a paradoxical increase in injury rates. This may be a particular concern in child and adolescent athletes in whom head injury rates are often higher than in adult athletes. ${ }^{64}$

\section{Medicolegal considerations}

Although agreement exists on the principal messages conveyed in this document, we acknowledge that the science of concussion is at an early stage, and therefore management and return to play decisions remain largely in the realm of clinical judgment on an individualised basis.

\section{Education}

As the ability to treat or reduce the effects of concussive injury after the event is minimal, education of athletes, colleagues, and the general public is a mainstay of progress in this field. Athletes and their healthcare providers must be educated about the detection of concussion, its clinical features, assessment techniques, and principles of safe return to play. Methods to improve education including web based resources, educational videos, and international outreach programmes such as Think First (www.thinkfirst.ca) are important in delivering the message. In addition, concussion working groups plus the support and endorsement of enlightened sport groups such as FIFA, IOC, and IIHF who initiated this endeavour have enormous value and must be pursued vigorously.

The promotion of fair play and respect for opponents are ethical values that should be encouraged in all sports and sporting associations. Similarly coaches, parents, and managers play an important part in ensuring that these values are implemented on the field of play.

\section{Research methods}

A number of research protocols and data evaluating concussion injury assessment, injury susceptibility, and brain function after injury were presented at both the Vienna and Prague conferences. Although they offer great potential for injury assessment, all of these techniques must be considered experimental at this time. Elite and professional teams are well placed to contribute to these efforts through athlete 
recruitment for studies showing the scientific value of such approaches.

Such research is essential in contributing to the science of concussion and will potentially provide valuable information for such important issues as clinical management, return to play guidelines, and long term outcome. Therefore research should be continued and encouraged, by both academics and sporting organisations.

\section{Future}

The issue of sports concussion management is continually evolving, and the usefulness of expert consensus in establishing a standard of care has been demonstrated by the Vienna agreement. The consensus group established at that meeting has provided continuing leadership in this field based on the initial mandate established at that time. ${ }^{1}$ We expect that this Prague agreement will be revised and updated at future meetings.

\section{Authors' affiliations \\ M Aubry, Chief Medical Officer, International Ice Hockey Federation R Cantu, Department of Surgery, Chief Neurosurgery Service and Director, Sports Medicine Service, Emerson Hospital, Concord, MA, USA; Medical Director, National Center for Catastrophic Sports Injury Research, Chapel Hill, NC, USA \\ J Dvorak, Chairman, FIFA Medical Research and Assessment Center (F-MARC); Chairman, Department of Neurology, Schulthess Clinic, Zurich, Switzerland}

T Graf-Baumann, Director, Office for Science Management, Administration and Scientific Director, German Society for MusculoSkeletal Medicine and Pain Therapy, FIFA Medical Research and Assessment Center (F-MARC), Teningen, Germany

K M Johnston, Neurosurgeon/Concussion Consultant, Departments of Neurosurgery, Kinesiology \& Physical Education, McGill University; Director, Concussion Program, McGill Sport Medicine Centre, Montreal, Canada

J P Kelly, Professor of Neurosurgery and Rehabilitation Medicine, University of Colorado School of Medicine, Denver, CO, USA M Lovell, Director, Sports Medicine Concussion Program, University of Pittsburgh; Co-director, National Hockey League Neuropsychology Program, Pittsburgh, PA, USA

P McCrory, Associate Professor, Center for Health, Exercise and Sports Medicine \& The Brain Research Institute, University of Melbourne, Melbourne, Australia

W Meeuwisse, Professor and Medical Director, University of Calgary Sport Medicine Centre; Sport Injury Epidemiologist, National Hockey League, Calgary, Alberta, Canada

P Schamasch, Medical and Scientific Director, International Olympic Committee, Lausanne, Switzerland

Competing interests: Dr Lovell is a shareholder of ImPACT, a neuropsychological testing programme. No other author has any declared conflict of interest or concussion industry affiliation.

This article will also be published in the April 2005 issues of the Clinical Journal of Sport Medicine, Physician and Sportsmedicine, and Neurosurgery.

\section{REFERENCES}

1 Aubry M, Cantu R, Dvorak J, et al. Summary and agreement statement of the first International Conference on Concussion in Sport, Vienna 2001. Phys Sportsmed 2002;30:57-62 (also co-published in Br J Sports Med 2002;36:3-7 and Clin J Sport Med 2002;12:6-12)

2 Congress of Neurological Surgeons. Committee on Head Injury Nomenclature: glossary of head injury. Clin Neurosurg 1966;12:386-94.

3 Johnston K, McCrory P, Mohtadi N, et al. Evidence based review of sportrelated concussion: clinical science. Clin J Sport Med 2001;11:150-60.

4 Hovda D, Lee S, Smith M, von Stuck S, et al. The neurochemical and metabolic cascade following brain injury: moving from animal models to man. J Neurotrauma 1995;12:903-6.

5 McIntosh TK, Smith DH, Meaney DF, et al. Neuropathological sequelae of traumatic brain injury: relationship to neurochemical and biomechanical mechanisms. Lab Invest 1996;74:315-42.

6 Cantu RC. Concussion severity should not be determined until all postconcussion symptoms have abated. Lancet 2004;3:437-8

7 Hinton-Bayre AD, Geffen G. Severity of sports-related concussion and neuropsychological test performance. Neurology 2002;59:1068-70.
$8 \mathrm{McC}$ rory PR, Ariens T, Berkovic SF. The nature and duration of acute concussive symptoms in Australian football. Clin J Sport Med 2000; 10:235-8.

9 Mrazik M, Ferrara MS, Peterson CL, et al. Injury severity and neuropsychological and balance outcomes of four college athletes. Brain Inj 2000;14:921-31.

10 Lovell MR, Collins MW, Iverson GL, et al. Recovery from mild concussion in high school atheltes. J Neurosurg 2003;98:296-301.

11 Collins MW, Grindel SH, Lovell MR, et al. Relationship between concussion and neuropsychological performance in college football players [see comments]. JAMA 1999;282:964-70.

12 Bleiberg J, Cernich A, Cameron K, et al. Duration of cognitive impairment after sports concussion. Neurosurgery 2004;54:1073-80.

13 McCrea M, Kelly J, Randolph C, et al. Immediate neurocognitive effects of concussion. Neurosurgery 2002;50:1032-42.

14 McCrory P, Johnston K, Meeuwisse W et al. Evidence based review of sport related concussion: basic science. Clin J Sport Med 2001;11:160-6.

15 Lovell M, Iverson G, Collins $M$, et al. Does loss of consciousness predict neuropsychological decrements after concussion. Clin J Sport Med 1999:9:193-8.

16 Cantu RC. Posttraumatic retrograde and anterograde amnesia: pathophysiology and implications in grading and safe return to play. J Athl Train 2001;36:244-8

17 Leninger B, Gramling S, Farrell A, et al. Neuropsychological deficits in symptomatic minor head injury patients after concussion and mild concussion. J Neurol Neurosurg Psychiatry 1990;53:293-6.

18 Yarnell P, Lynch S. The 'ding': amnestic state in football trauma. Neurology 1973;23:196-7.

19 Yarnell PR, Lynch S. Retrograde memory immediately after concussion. Lancet 1970;1:863-4.

20 McCrory P, Collie A, Anderson V, et al. Can we manage sport-related concussion in children the same as adults? Br J Sports Med 2004;38:516-19.

21 Gusckiewicz K, Bruce S, Cantu R, et al. Recomendations on management of sport-related concussion: summary of the national Athletic Trainers' Association position statement. Neurosurgery 2004;55:891-2

22 McCrory P. Preparticipation assessment for head injury. Clin J Sport Med 2004;14:139-44.

23 Johnston KM, Lassonde M, Ptito A. A contemporary neurosurgical approach to sport-related head injury: The McGill concussion protocol. J Am Coll Surg 2001:515-24.

24 Delaney JS, Lacroix VJ, Leclerc S, et al. Concussions during the 1997 Canadian Football League season. Clin J Sport Med 2000;10:9-14.

25 Delaney J, Lacroix V, Leclerc S, et al. Concussions among university football and soccer players. Clin J Sport Med 2002;12:331-8.

26 Iverson G, Gaetz $M$, Lovell $M$, et al. Relation between subjective fogginess and neuropsychological testing following concussion. J Int Neuropsychol Soc 2004; 10:904-6.

27 Maddocks DL, Dicker GD, Saling MM. The assessment of orientation following concussion in athletes. Clin J Sport Med 1995;5:32-5.

28 McCrea M, Kelly J, Randolph C, et al. Standardised assessment of concussion (SAC): on site mental status evaluation of the athlete. J Head Trauma Rehabil 1998; 13:27-35.

29 McCrea M, Kelly JP, Kluge J, et al. Standardized assessment of concussion in football players. Neurology 1997;48:586-8.

$30 \mathrm{McCrory} \mathbf{P}$. Videoanalysis of the acute clinical manifestations of concussion in Australian rules football. In: SMA/ACSP Annual Scientific Conference; 1996. Canberra: SMA, 1996:214-15.

31 McCrory $\mathbf{P}$, Berkovic $\mathrm{S}$. Videoanalysis of the motor and convulsive manifestations of concussion in acute sport related injury. Neurology 2000;54: 1488-92.

32 Kelly J, Rosenberg J. Diagnosis and management of concussion in sports. Neurology 1997:48:575-80.

33 McCrea M, Randolph C, Kelly J. In: The standardized assessment of concussion (SAC): manual for administration, scoring and interpretation, 2nd ed. Waukesha, WI: CNS Inc, 2000

34 Turner M. Concussion and head injuries in horse racing. In: Turner $M$, ed. The Jockey Club conference on head injury in sport, 1998. London: The Jockey Club of England, 1998.

35 Johnson K, McCrory P, Mohtadi N, et al. Evidence-based review of sportsrelated concussion: clinical science. Clin J Sport Med 2001;11:150-9.

36 Grindel S, Lovell M, Collins M. The assessment of sport-related concussion: the evidence behind neuropsychological testing and management. Clin J Sport Med 2001;11:134-44.

37 Collie A, Merouf P, Darby D. Computerised neuropsychological testing in sport. Br J Sports Med 2001;35:in press.

38 Guskiewicz K. Postural stability assessment following concussion. Clin J Sport Med 2001;11:182-90.

39 Johnston K, Ptito A, Chankowsky J, et al. New frontiers in diagnostic imaging in concussive head injury. Clin J Sport Med 2001;11:166-76.

40 Chen J, Johnston K, Frey S, et al. Functional abnormalities in symptomatic concussed athletes: an fMRI study. Neuroimage 2004;22:68-82.

41 Kelly J, Jantzen B, Steinberg F, et al. A prospective functional MR imaging study of mild traumatic brain injury in college football players. Am J Neuroradiol 2004;25:738-45.

42 Teasdale G, Nicol J, Murray G. Association of Apolipoprotein E polymorphism with outcome after head injury. Lancet 1997;350:1069-71.

43 Friedman G, Froom P, Sazbon L, et al. Apoliporotein E-epsilon 4 genotype predicts a poor outcome in survivors of traumatic brain injury. Neurology 1999:52:244-8.

44 Gross R. APOE epsilon4 allele and chronic traumatic brain injury. JAMA 1997;278:2143. 
45 Katzman R, Galasko DR, Saitoh T, et al. Apolipoprotein-epsilon4 and head trauma: synergistic or additive risks? [letter; comment]. Neurology 1996;46:889-91.

46 Liberman J, Stewart W, Wesnes K, et al. Apolipoprotein E e4 and short term recovery from predominantly mild brain injury. Neurology 2002;58: 1038-44.

47 Nicoll JA, Roberts GW, Graham DI. Apolipoprotein E epsilon 4 allele is associated with deposition of amyloid beta-protein following head injury [see comments]. Nat Med 1995;1:135-7.

48 Nicoll JA, Roberts GW, Graham DI. Amyloid beta-protein, APOE genotype and head injury. Ann N Y Acad Sci 1996;777:271-5.

49 Jordan B, Relkin N, Ravdin L. Apolipoprotein E epsilon 4 associated with chronic traumatic brain injury in boxing. JAMA 1997;278:136-40.

50 Kors $\mathbf{E}$, Terwindt $G$, Vermeulen $F$, et al. Delayed cerebral edema and fatal coma after minor head trauma: role of the CACNA1A calcium channel subunit gene and relationship with familial hemiplegic migraine. Ann Neurol $2001 ; 49: 753-60$.

51 Dupuis $F$, Johnston $K$, Lavoie $M$, et al. Concussion in athletes produces brain dysfunction as revealed by event related potentials. Clin J Sport Med 2005; in press.

52 Gaetz M, Goodman D, Weinberg H. Electrophysiological evidence for the cumulative effects of concussion. Brain Inj 2000;14:1077-88.

53 Lavoie M, Dupuis F, Johnston K, et al. Visual p300 effects beyond symptoms in concussed college athletes. J Clin Exp Neuropsychol 2004;26:55-73.

54 Hinton-Bayre AD, Geffen G, McFarland K. Mild head injury and speed of information processing: a prospective study of professional rugby league players. J Clin Exp Neuropsychol 1997;19:275-89.
55 Clark CR, O'Hanlon AP, Wright MJ, et al. Event-related potential measurement of deficits in information processing following moderate to severe closed head injury. Brain inj 1992;6:509-20.

56 Cremona-Meytard SL, Geffen GM. Visuospatial attention deficits following mild head injury in Australian rules football players. In: Hendy J, Caine D, Pfaff A, et al, eds. The life cycle: development, maturation, senescence. Proceedings of the 16th Annual Brain Impairment Conference, 1993. Sydney: Australian Academic Press, 1993:137-47.

57 Cremona-Meytard SL, Clark CR, Wright MJ, et al. Covert orientation of visual attention after closed head injury. Neuropsychologia 1992;30:123-32.

58 Igebrigtsen T, Romner B, Trumpy JH. Management of minor head injury: the value of early computed tomography and serum protein S-100 measurements. J Clin Neurosci 1997;4:29-34.

59 Otto $M$, Holthusen S, Bahn $E$, et al. Boxing and running lead to a rise in serum levels of S-100B protein. Int J Sports Med 2000;21:551-5.

60 Johnston K, Bloom G, Ramsay J, et al. Current concepts in concussion rehabilitation. Curr Sports Med Rep 2004;3:316-23.

61 McCrory P. Should we treat concussion pharmacologically? Br J Sports Med 2002;36:3-6.

62 Bloom G, Horton A, McCrory $P$, et al. Sport psychology and concussion: new impacts to explore. Br J Sports Med 2004;38:519-21.

63 Hagel B, Meeuwisse W. Risk compensation: a "side effect" of sport injury prevention? Clin J Sport Med 2004;14:193-6.

64 Orchard J, Wood T, Seward H, Broad A. Comparison of injuries in elite senior and junior Australian football. J Sci Med Sport 1998;1:83-8.

\section{$\mathrm{ECHO}$}

\section{Human articular cartilage fails to respond to physical training}

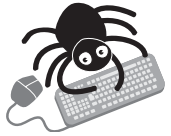

Please visit the British Journal of Sports Medicine website [www. bjsportmed. com] for a link to the full text of this article. $\mathrm{n}$ in vivo study has suggested that the amount human articular cartilage deforms after
exercise is independent of physical training, as shown by responses of patellar and
tibial cartilage and between professional athletes and others. Deformity of patellar cartilage ranged from $-5.9 \%$ to $-2.8 \%$, in a dose dependent manner, for various activities in 12 healthy young volunteers $(-5.9 \%$ knee bends, $-5.0 \%$ running, $-4.7 \%$ squatting, $-4.5 \%$ cycling, and $+2.8 \%$ walking). Deformity of femorotibial cartilage, however, was greatest for high impact loading —as in jumps from $40 \mathrm{~cm}$ height (-7\%) — but small for other activities. There was no significant difference in deformity of patellar cartilage in weightlifters $(-2.9 \%)$, bobsleigh sprinters $(-3.9 \%)$, or non-athletes $(-4.1 \%)$.

Deformity was measured by magnetic resonance imaging and three dimensional image analysis before and after exercise. Of the 50 volunteers, six men and six women did patellar exercises and five men and five women femorotibial exercises (knee bends, (one leg) static loading of weight, and jumps from $40 \mathrm{~cm}$ height onto one leg. Seven weightlifters and seven bobsleigh sprinters were compared with 14 healthy young men for deformity of patellar cartilage induced by knee bends.

This is the first in vivo study of human patellar and femorotibial cartilage response to such a wide range of physical activities. The authors hypothesised from animal studies that physical training would result in less deformity, but this seems not to be true for humans. Understanding how cartilage behaves in vivo is important for understanding the process of osteoarthritis and the local environment of transplanted cartilage.

A Eckstein F, et al. Annals of the Rheumatic Diseases 2005;64:291-295. 